\title{
Relationship Between Small Bowel Obstruction and Small Bowel Feces Sign: Four Cases Report
}

\author{
Fatih Altintoprak ${ }^{\mathrm{a}, \mathrm{e}}$, Yasemin Gunduz ${ }^{\mathrm{b}}$, Omer Yalkin ${ }^{\mathrm{c}}$, Kemal Gundugdu ${ }^{\mathrm{c}}$, \\ Serbulent Gokhan Beyaz ${ }^{\mathrm{d}}$, Osman Nuri Dilek ${ }^{\mathrm{a}}$
}

\begin{abstract}
Small bowel obstruction is a commonly encountered clinical condition in emergency departments. Small bowel feces sign (SBFS) is a CT finding that can be detected in small bowel obstruction. SBFs was identified in the abdominal CT examinations of four patients admitted to the emergency department with complaints of abdominal pain and vomiting. Two patients were treated by surgery and two with medication. In these cases were presented to highlight the importance of the SBFs in the treatment algorithm.
\end{abstract}

Keywords: Intestinal obstruction; Small-bowel feces; CT

\section{Introduction}

Mechanical small bowel obstruction (SBO) is the most commonly observed surgical disorder of the small intestine. Intra-abdominal adhesions associated with previous abdominal surgery are responsible for up to $75 \%$ of cases with small bowel obstruction. In very rare cases, metastatic malignancies, diverticulitis (non-Meckel's) and bezoars can also cause SBO. In SBO, proper identification of the etiology and the level of obstruction are very important for clinicians, as this provides directions for the treatment algorithm (op-

\footnotetext{
Manuscript accepted for publication August 5, 2013

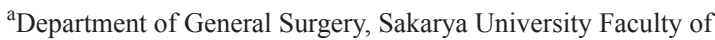
Medicine, Sakarya, Turkey

${ }^{\mathrm{b}}$ Department of Radiology, Sakarya University Faculty of Medicine, Sakarya, Turkey

${ }^{c}$ Department of General Surgery, Sakarya University Research and Educational Hospital, Sakarya, Turkey

${ }^{\mathrm{d}}$ Department of Anesthesiology, Sakarya University Faculty of Medicine, Sakarya, Turkey

${ }^{\mathrm{e}}$ Corresponding author: Fatih Altintoprak, Department of General Surgery, Sakarya University Faculty of Medicine, Sakarya, Turkey. Email: fatihaltintoprak@yahoo.com
}

doi: http://dx.doi.org/10.4021/jmc1429e erative or non-operative treatment).

Abdominal radiographic findings specific for SBO are dilated small bowel loops, air-fluid levels and paucity of air in the colon. Although the sensitivity of abdominal radiographs in the detection of SBO ranges from 70 to $80 \%$, the specificity is lower. Computerized tomography (CT) has proven to be an excellent and widely used method for the diagnosis of SBO. The CT scanning has a sensitivity of 80 to $90 \%$ and a specificity of 70 to $90 \%$ in the detection of SBO. The CT scanning also provides a general evaluation of the abdomen and may therefore reveal the etiology of the obstruction [1]. In this manuscript, the characteristic appearances of small bowel feces sign (SBFs) are presented and the clinical significance is discussed.

\section{Case Report}

\section{Case 1}

A 74-year-old female patient was admitted to the emergency department with abdominal pain, distension and vomiting

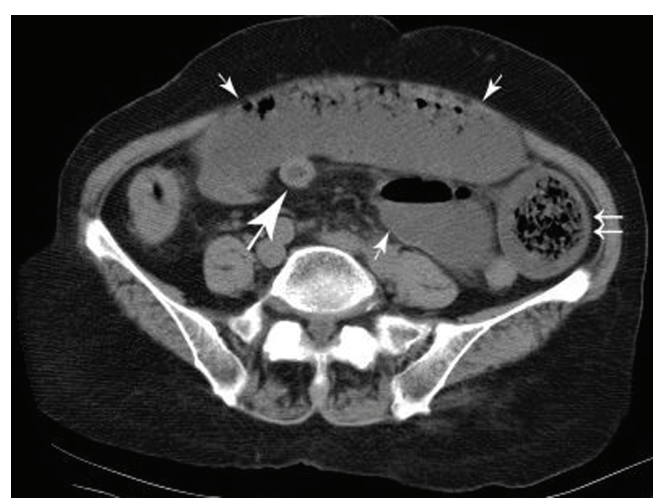

Figure 1. Abdominal CT examination (axial section), circular wall thickening at the level of jejuno-ileal segment of the small intestine, leading to a narrowing of the lumen (large arrow). The intestinal dilatation ends at this level. SBF can be observed in the immediately proximal jejunum segment (double arrow) along with the fluid levels in the more proximal segments (small arrows). 


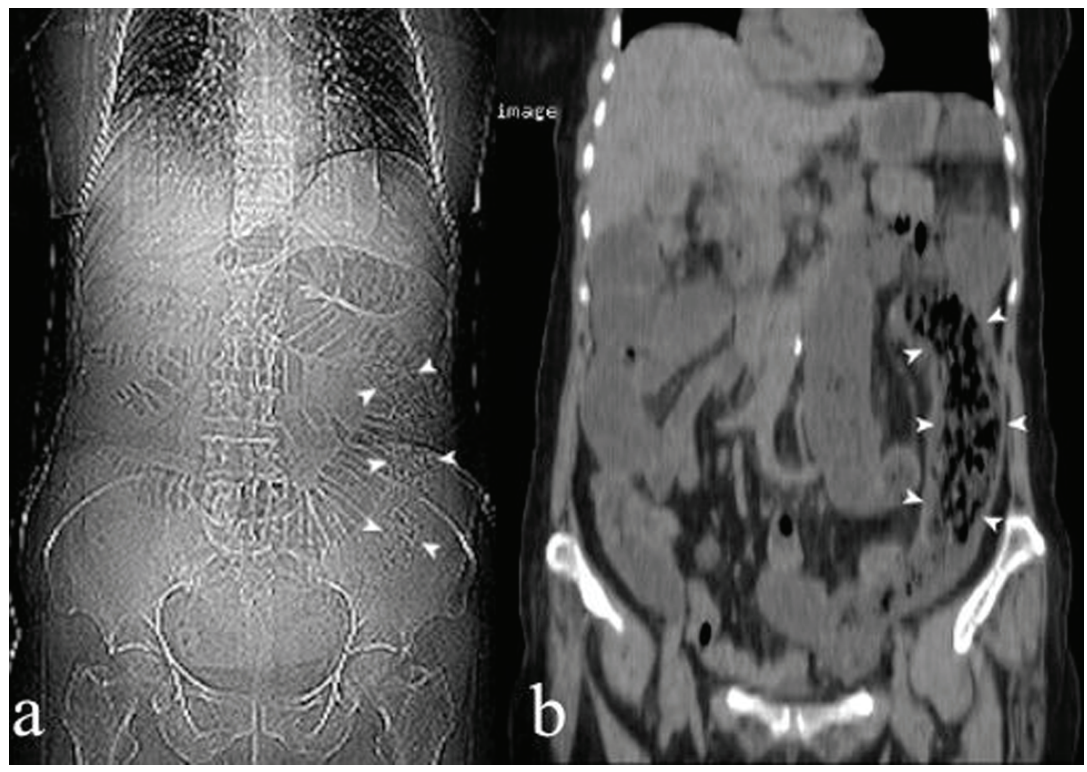

Figure 2. Plain abdominal X-ray (a) and coronal reconstruction of abdominal CT image (b); for SBF extending through a length of $20-25 \mathrm{~cm}$ in the left quadrant of the abdomen at the level of the jejunal segment (arrow heads).

complaints, all of which were ongoing for one month and had worsened over the past 3 days. The patient experienced significant weight loss $(8 \mathrm{~kg})$ over the last 6 months, and had no history of previous abdominal surgeries. Abdominal CT showed a severe dilatation of the jejunal segments (diameter: $5.3 \mathrm{~cm}$ ) with a normal diameter of the distal segments. A detailed examination revealed circular wall thickening at the jejuno-ileal level, causing a narrowing of the lumen. The intestinal dilatation ended in this region. SBFs was visualized in the dilated small intestine segment at $1-2 \mathrm{~cm}$ proximal to the obstruction region and extending to $20-25$ $\mathrm{cm}$ proximal. Within the more proximally dilated segments, only the presences of fluids were observed (Fig. 1, 2). The patient underwent emergency surgery with a diagnosis for mechanical intestinal obstruction, and the abdominal exploration revealed an obstructive circular wall thickening in the small bowel segment $130 \mathrm{~cm}$ distal to the ligament of Treitz. In the segment $20-25 \mathrm{~cm}$ proximal to the obstruction, an accumulation of the small bowel contents was observed while the more proximal segments were filled with only fluid. A segmental resection of the small intestine with an end jejunostomy was performed. The patient was discharged on the fourth day following surgery as uneventful. Histopathological examination revealed metastatic carcinoma of the small

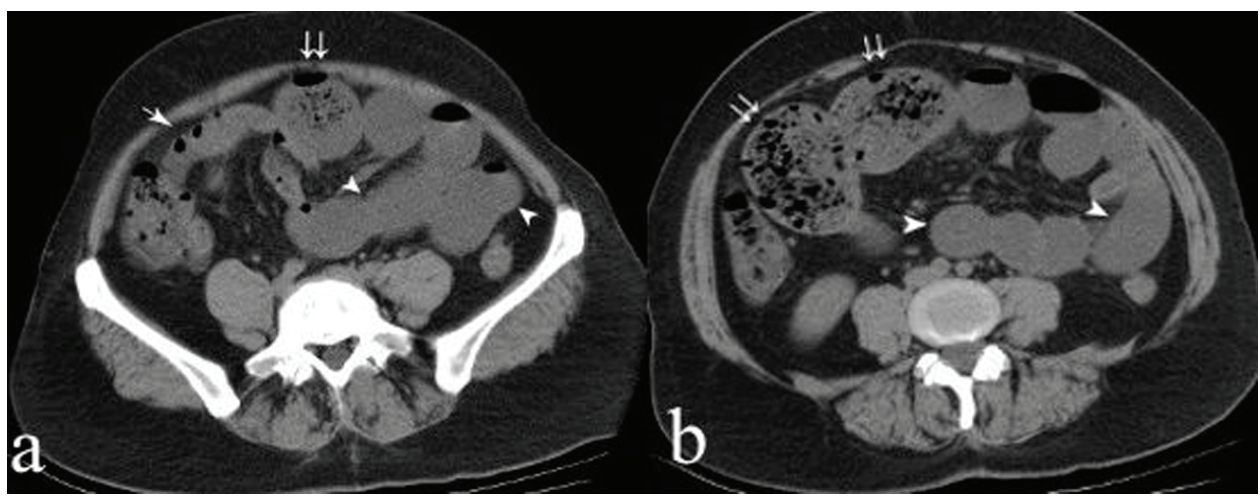

Figure 3. Abdominal CT examination (axial section): a) the terminal ileum segment has a normal diameter (arrow) while the proximal small bowel segments appear as dilated. SBF is present in the dilated segments immediately proximal to the terminal ileum (double arrow), while only fluids are present in the more proximal dilated segments (arrow heads). b) SBF appearance (double arrows) and proximally dilated small bowel segments filled only with fluid (arrow heads). 


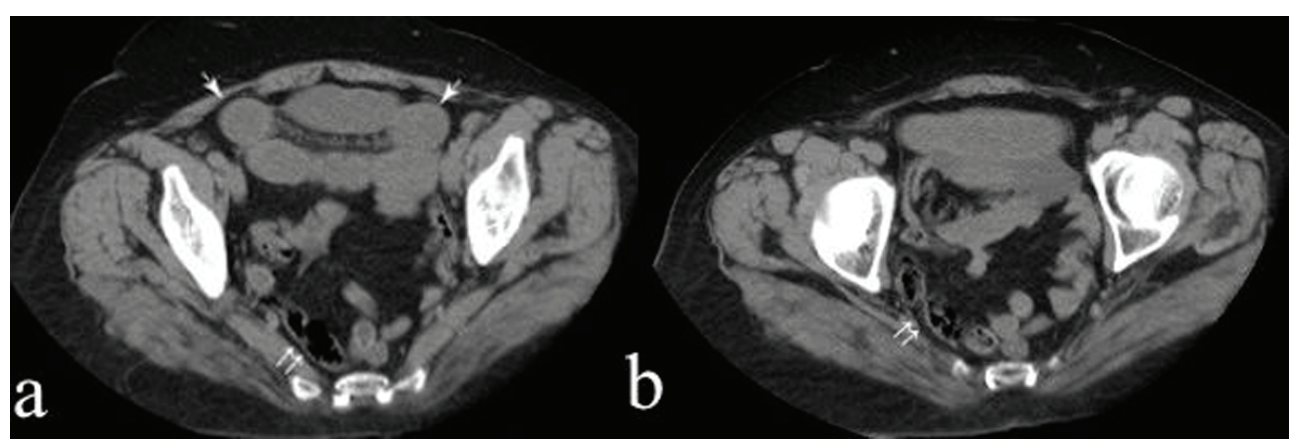

Figure 4. Abdominal CT examination (axial section); a) SBF appearance extending to the level of the terminal ileum (double arrow), with mild dilation of the more proximal segments (arrows). b) SBF extending to the level of the terminal ileum (double arrow).

intestine. The patient died on the 15th day following, before further investigations could be performed.

\section{Case 2}

A 37-year-old female was admitted to the emergency department with increasing abdominal pain, bloating, and vomiting complaints, ongoing for one week. The patient had occasionally experienced these complaints within the past 2 years, and had no history of previous abdominal surgeries. Abdominal CT revealed a severe dilatation of small bowel segments (diameter: $6.4 \mathrm{~cm}$ ) towards the terminal ileum, while the bowel diameter at the level of the terminal ileum was determined as being normal (Fig. 3). SBFs was visualized at $5 \mathrm{~cm}$ proximal to the obstruction, extending over a distance of $20-25 \mathrm{~cm}$ proximally. No specific cause of obstruction could be determined. The patient underwent emergency surgery with mechanical intestinal obstruction diagnosis, and abdominal exploration revealed a diverticulum on the antimesenteric wall $140 \mathrm{~cm}$ distal to the ligament of Treitz with a diameter of approximately $7-8 \mathrm{~cm}$, which caused torsion of the small bowel due to adherence by distal segments of the small intestine. Segmental resection of the small bowel including the diverticulum, and end-to-end anastomosis was performed. The patient was discharged five days after surgery as uneventful. The histopathological examination of the small intestine revealed diverticulitis.

\section{Case 3}

A 56-year-old female patient was admitted to the emergency department with abdominal pain and vomiting complaints for one day. The patient had a history of previous abdominal surgery due to peptic ulcer perforation. Abdominal CT findings consisted of mild dilatation in the segments of the jejunum (diameter: $2.7 \mathrm{~cm}$ ). At the level of the terminal ileum, SBFs was identified in the $10 \mathrm{~cm}$ segment where the small bowel dilatation ended (Fig. 4). However, there no specific pathology could be determined as the cause of obstruction. As there were no signs of acute abdominal syndrome and no severe dilation of the small intestines identified by abdominal CT, the patient was treated medically (cessation of food intake orally and nasogastric decompression). The patient started feeding orally on the 4th day of clinical follow-up, and was discharged from the hospital on the 6th day. Currently, the patients is still is symptom-free at the 7 th month

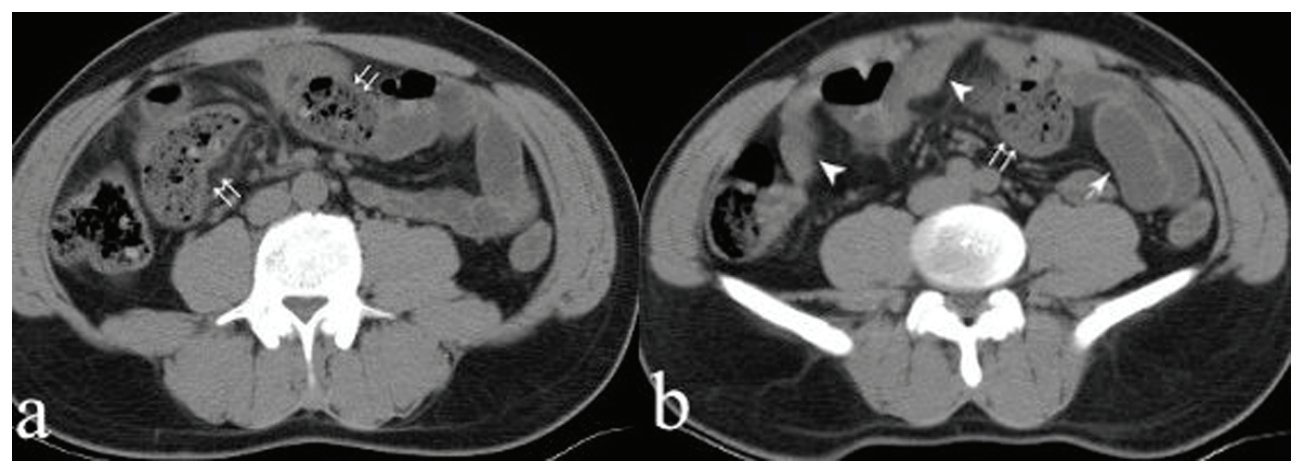

Figure 5. Abdominal CT examination (axial section); a) SBF can be observed in the ileum segment (double arrows). b) the terminal ileum segment and distal segment to the obstruction site has a normal diameter (arrow heads) while the proximal small bowel segments appear as mild-dilated and filled only with fluid (arrow). 
of follow-up.

\section{Case 4}

A 36-year-old man patient was admitted to the emergency department with the complaints of abdominal pain and vomiting for two days. The patient had a history of previous abdominal surgery due to traumatic diaphragma injury. Abdominal CT findings consisted of mild dilatation of the ileum segments (diameter: $2.5 \mathrm{~cm}$ ) with a normal diameter of the distal segments. SBFs was visualized in the dilated small intestine segment (Fig. 5). No specific cause of obstruction could be determined. The patient was treated medically. The patient started feeding orally on the 3th day of clinical follow-up, and was discharged from the hospital on the 5th day. Currently, the patients is still is symptom-free at the 3th month of follow-up.

\section{Discussion}

While definite diagnosis for SBO required surgical intervention in past years, nowadays the etiologic causes are easily determined especially with the advent of routine abdominal $\mathrm{CT}$ examination, and the patients are spared from unnecessary surgical interventions. CT findings for small bowel obstruction includes a discrete transition zone with a proximal dilation of the bowel along with distal decompression, the appearance of intraluminal contrast that does not pass beyond the transition zone and a colon containing little gas or fluid. The CT scans may also provide evidence for the presence of closed-loop obstruction/strangulation, and offers a general evaluation of the abdomen [2].

Small BFs were originally described as the presence of gas bubbles in particulate matter in dilated segments of small bowels. These symptoms are probably caused by stasis within the obstructed loop, which allows more time for fluid absorption across the intestinal wall and accumulation of undigested food particles [3]. The SBFs detection rates in the literature vary between 4 to $56 \%[3,4]$. The reason for this variability in incidence may be due to the different design of the studies in the literature, for example, the evaluation of the CT findings only without establishing any correlation with clinical course or operative findings, the examination of cases of SBO due to specific causes, and the evaluation of the CT findings of all patients admitted by emergency departments with complaints of abdominal pain.

The appearance of SBF along with SBO often provides an indication of the severity of obstruction and the time of occurrence. Patients without obstruction have also been reported. Lazarus et al [3] have determined that SBFs presence was more frequent in moderate and high-grade SBO in comparison to low-grade SBO. Catalano [5] has stated that 1 out of 7 patients with SBFs were diagnosed with an acute onset of clinical symptoms due to strangulation, while the remaining 6 patients had progressive obstruction. Both of cases (case 1 and 2) had a chronic course accompanied by acute exacerbations of clinical symptoms and were eventually treated by surgery. The CT examination revealed signs of high-grade SBO in both cases.

In a group of 1642 patients with abdominal CT performed due to abdominal pain, Jacobs et al [6] have evaluated 100 patients with SBFs. Only $32(32 \%)$ of the patients were clinically diagnosed as having SBO, while the remaining $68(68 \%)$ patients were reported as having non-obstructive pathology. In the same study, it was emphasized that the presence of SBFs could serve as diagnosis of SBO only if the SBFs were associated with moderate or severe small bowel dilatation. Furthermore, it was stated that the presence of SBFs is not sufficient for deciding whether an operative treatment will be followed for patients with $\mathrm{SBO}[7,8]$; however, it is very useful in determining the level of obstruction as it is located proximally to the obstruction [3]. In our medically treated cases (case 3 and 4), the clinical symptoms were acute from the onset, and they have mild SBO signs on CT.

Another aspect to be considered for differential diagnosis of SBFs is bezoars, which is a rare cause of SBO. However, they are encountered more frequently in certain regions, especially in regions where certain nutrients (Persimmon) are commonly consumed. The general and pathognomonic CT findings of bezoars have been described in the literature. An intraluminal mass appearance at the obstruction site that is round or oval shaped, with a mottled gas pattern, associated with dilated proximal intestinal segments and collapsed distal segments are the CT findings for small intestinal bezoars [9]. There are notable differences between SBFs and bezoar: small-bowel feces yield an elongated form of greater length and less compact nature than bezoars, more amorphous and affect longer segments than bezoars. Furthermore, small-bowel feces are usually present in dilated small-bowel loops, while small-bowel bezoars are located at the transition zone between dilated and collapsed small-bowel loops, and SBFs tend to be more tubular in shape $[4,10]$. Since persimmon grows naturally in our region, bezoars are commonly encountered in our clinical practice. However, CT findings of all four patients were different from those associated with bezoars, instead bearing similarity to the characteristics of SBFs described above (located proximal to the obstruction, with a segmental involvement of at least $10 \mathrm{~cm}$, tubular and intraluminal mottled gas pattern that are less compact than bezoars).

In conclusion, although SBFs indicate the presence of mechanical SBO, these symptoms are neither sensitive nor specific for the diagnosis of SBO. It may be useful in the diagnosis of SBO only when associated with moderate or severe small bowel dilatation. Furthermore, as it indicative of the obstruction's location, it can also assist clinicians in determining the etiology of the obstruction and the treatment 
method.

\section{Disclosure}

This study is presented at XVI. Annual Meeting of European Society of Surgery (ESS) Istanbul, 2012 as poster presentation.

\section{References}

1. Maglinte DD, Heitkamp DE, Howard TJ, Kelvin FM, Lappas JC. Current concepts in imaging of small bowel obstruction. Radiol Clin North Am. 2003;41(2):263-283, vi.

2. Balthazar EJ, Liebeskind ME, Macari M. Intestinal ischemia in patients in whom small bowel obstruction is suspected: evaluation of accuracy, limitations, and clinical implications of CT in diagnosis. Radiology. 1997;205(2):519-522.

3. Lazarus DE, Slywotsky C, Bennett GL, Megibow AJ, Macari M. Frequency and relevance of the "small-bowel feces" sign on CT in patients with small-bowel obstruction. AJR Am J Roentgenol. 2004;183(5):1361-1366.

4. Kim JH, Ha HK, Sohn MJ, Kim AY, Kim TK, Kim
PN, Lee MG, et al. CT findings of phytobezoar associated with small bowel obstruction. Eur Radiol. 2003;13(2):299-304.

5. Catalano O. The faeces sign. A CT finding in small-bowel obstruction. Radiologe. 1997;37(5):417-419.

6. Jacobs SL, Rozenblit A, Ricci Z, Roberts J, Milikow D, Chernyak V, Wolf E. Small bowel faeces sign in patients without small bowel obstruction. Clin Radiol. 2007;62(4):353-357.

7. Deshmukh SD, Shin DS, Willmann JK, Rosenberg J, Shin L, Jeffrey RB. Non-emergency small bowel obstruction: assessment of CT findings that predict need for surgery. Eur Radiol. 2011;21(5):982-986.

8. Zielinski MD, Eiken PW, Bannon MP, Heller SF, Lohse CM, Huebner M, Sarr MG. Small bowel obstructionwho needs an operation? A multivariate prediction model. World J Surg. 2010;34(5):910-919.

9. Zissin R, Osadchy A, Gutman V, Rathaus V, ShapiroFeinberg M, Gayer G. CT findings in patients with small bowel obstruction due to phytobezoar. Emerg Radiol. 2004;10(4):197-200.

10. Delabrousse E, Lubrano J, Sailley N, Aubry S, Mantion GA, Kastler BA. Small-bowel bezoar versus smallbowel feces: CT evaluation. AJR Am J Roentgenol. 2008;191(5):1465-1468. 